\title{
Generación de viajes de emprendimientos residenciales: estudio de casos en la ciudad de Córdoba, Argentina
}

\author{
Lucía Bordese ${ }^{1}$ y Jorge José Galarraga ${ }^{2}$
}

\begin{abstract}
Resumo: Se estima que aproximadamente el $80 \%$ de todos los viajes que se realizan en una ciudad tienen su origen o destino en el hogar. Como consecuencia el conocimiento de la generación de este tipo de viajes resulta de la mayor importancia para avanzar hacia una movilidad urbana sustentable. Este trabajo considera dos ambientes bien diferenciados con sus modelos y tasas de generación. Por un lado se estudian establecimientos residenciales de baja densidad, constituidos por casas aisladas y con escaso acceso al servicio de transporte público. Por otro lado se estudian complejos de departamentos, con alta densidad, mejor acceso al servicio de transporte público y mayor diversidad de uso del suelo circundante. En base al estudio de 9 casos en la ciudad de Córdoba, Argentina, se ha caracterizado la generación de viajes en auto y a pie, obteniendo importantes diferencias entre los dos ambientes estudiados.
\end{abstract}

Palabras-claves: tasas de generación, emprendimientos residenciales, movilidad urbana sustentable.

\begin{abstract}
It is estimated that approximately $80 \%$ of all trips made in a city have their origin or destination in the home. Therefore, knowledge of the generation of this type of travel is important to move towards a sustainable urban mobility. The paper considers two different environments with their models and rates of generation: low-density residential facilities, consisting of isolated houses and with little access to public transport, and apartments with high density, better access to public transport and more diverse use of the soil. Based on the study of 9 cases in the city of Cordoba, Argentina, it has been characterized the generation of travel by car and on foot, obtaining important differences between the two environments studied.
\end{abstract}

Keywords: generation rates, residential facilities, sustainable urban mobility.

\section{INTRODUCCIÓN}

Un fenómeno cada vez más frecuente en las ciudades del mundo y que ha llevado a un cambio en la estructura de las mismas es la dispersión urbana, producida por el movimiento de la población desde el centro de la ciudad a las zonas urbanas periféricas. Dicho fenómeno se traduce en desarrollos de baja densidad y menor accesibilidad que son más difíciles y caros para servir por modos de transporte más eficientes, lo que trae como consecuencia una alta dependencia del vehículo particular (La Paix et al, 2012), produciendo mayor congestión, incremento de los tiempos de viaje y negativos impactos ambientales, sociales y económicos. La ocupación desordenada del suelo debido a la falta de un plan de ordenamiento urbanístico a largo plazo, la falta de articulación con un plan de infraestructura y transporte, o bien la falta de mecanismos de control para hacer cumplir dichos planes cuando existen, es un problema común en las ciudades latinoamericanas (Portugal et al, 2010).

Los Polos Generadores de Viajes (PGV) son concentradores de actividades en el espacio, y se caracterizan por impactar en la accesibilidad y el desempeño de los sistemas de transporte y viarios, y por promover los desplazamientos en el desenvolvimiento urbano. Y en función de esa potencialidad de producir externalidades es fundamental que sean adecuadamente planeados, localizados y dimensionados,

\footnotetext{
1 Lucía Bordese, Departamento de Ingeniería de Transporte, UNC. (lubordese@hotmail.com)

2 Jorge José Galarraga, Departamento de Ingeniería de Transporte, UNC. (jorgala@efn.uncor.edu)
}

Manuscrito recebido em 29/03/2016 e aprovado para publicação em 19/10/2016

Este artigo é parte de TRANSPORTES v. 25, n. 1, 2017. ISSN: 2237-1346 (online). DOI:10.14295/transportes.v25i1.1105 para lo cual es necesario conocer las especificidades locales en que se insertan (Portugal, 2012).

Los shoppings, hipermercados, hospitales, universidades, estadios, establecimientos residenciales, hoteles, estaciones de transporte público, son algunos tipos de PGV (Portugal y Goldner, 2003). En este estudio, nos centraremos en los establecimientos residenciales, donde se estima que cerca del $80 \%$ de todos los viajes tienen su origen o destino (Ortúzar y Willumsen, 2011), y sin embargo, la previsión de la generación de viajes en este tipo de emprendimientos está poco estudiada a nivel local. La dificultad para estudiar este tipo de uso radica en la diversidad en que ocurren. Cada forma de ocupación, como así también, cada perfil de residentes, presenta diferentes tasas de generación de viajes e impactos en el sistema vial asociado. En la tasa de generación de viajes, como en la elección modal, influyen dos tipos de factores principalmente: los socioeconómicos y los de localización.

El objetivo general de este estudio es comprender y modelar la generación de viajes de unidades residenciales de la Ciudad de Córdoba, desde la perspectiva de la movilidad urbana sustentable.

Se plantean así los siguientes objetivos específicos:

a) Caracterizar dos tipos diferentes de unidades residenciales, uno de baja densidad, deficiente acceso al servicio público de transporte y uso del suelo exclusivamente residencial; y otro de alta densidad, aceptable acceso al servicio público de transporte y con diversidad en el uso del suelo adyacente.

b) Estimar las tasas de generación de viajes a pie y en auto para las horas pico de la tarde y de la mañana, como así también valores diarios.

c) Estimar los modelos de generación de viajes que resulten más adecuados.

d) Comparar los resultados obtenidos con otras tasas y modelos disponibles. 
Se entiende por desarrollo sostenible al desarrollo que satisface las necesidades de las generaciones presentes sin comprometer las posibilidades de las generaciones futuras para satisfacer sus propias necesidades. Pero es importante además, entender que el desarrollo sostenible debe apoyarse sobre tres pilares: el progreso económico, la justicia social y la preservación del medio ambiente. De esta forma, un modelo de movilidad urbana sustentable debe asegurar la protección del medio ambiente, mantener la cohesión social y la calidad de vida de los ciudadanos y favorecer el desarrollo económico.

\section{ANTECEDENTES}

Los estudios sobre Polos Generadores de Viaje se remontan a la década de 1950 y de forma más sistemática a partir de 1970 con respaldo técnico de organismos internacionales, en especial del Institute of Transportation Engineers (ITE) de Estados Unidos. En un comienzo, se estudiaban los impactos que estos polos generaban a la circulación y al estacionamiento de los vehículos. Con el tiempo, otros impactos comenzaron a percibirse y valorarse tales como los producidos en el transporte público, en el transporte de cargas y en los modos no motorizados.

El ITE ha desarrollado un manual que provee tasas de generación de viajes y modelos para diversos usos de suelo. Por lo general, los datos utilizados para fijar estas tasas se han extraído de zonas suburbanas, las cuales poseen estacionamiento gratuito y abundante, baja densidad, usos de suelo individuales, y mínimos servicios de transporte. Numerosos estudios (Handy et al., 2013; Arrington y Cervero, 2008; entre otros) indican que estas tasas a menudo sobreestiman significativamente el número de viajes de los automóviles para proyectos ubicados en zonas urbanas cerca del transporte y a poca distancia a pie de otros usos de suelo.

En este marco, y en referencia al fenómeno de dispersión urbana, algunos estudios buscan conocer la relación entre el entorno construido (analizando variables tales como diversidad de uso de suelo, accesibilidad, densidad residencial, y diseño de la red local de calles) y el comportamiento de los viajes. Dentro de estos estudios pueden citarse un estudio realizado en California (Handy et al., 2013) sobre tasas de generación de viaje multimodales para proyectos denominados de crecimiento inteligente (ubicados en zonas urbanas, cercanos al transporte público y a poca distancia a pie de otros usos de suelo), el estudio realizado por Arrington y Cervero (2008) sobre Desarrollos Orientados al Transporte Público TOD (proyectos multifamiliares de vivienda de diferentes tamaños cerca de estaciones de transporte ferroviario), el estudio realizado por el Departamento de Transporte del Estado de California (Kimley-Horn et al, 2008) que estudia la densificación de ambientes urbanos, a partir de los Desarrollos de Relleno (nuevos proyectos de desarrollo y de reconstrucción situados en terrenos baldíos o subutilizados en las áreas desarrolladas existentes), el estudio en Carolina del Norte (Khattak y Rodríguez, 2005), sobre el comportamiento de los viajes en barrios Neo-Tradicionales (caracterizados por tener densidades superiores de desarrollo, alta conectividad de calles y aceras continuas, combinadas con usos de suelo mezclados), el estudio realizado en el norte de California por Handy et al (2005), o bien, algunos estudios realizados en América del Sur, como los realizados en Brasil, ciudad de Niteroi, por Grieco (2010) y por el equipo técnico de Nittrans (2011), o el estudio de tasas de generación de viajes en Mérida, Venezuela, de Angulo y Guerrero (2008).

Algunos estudios tienen como objetivo principal encontrar correlaciones entre el entorno construido y la elección del modo con el fin de saber qué variables pueden aumentar el uso del transporte público, como es el caso de un estudio realizado en Italia, en la ciudad de Roma por Gori et al (2013), o el artículo presentado por Tsai (2012) realizado para el Área Metropolitana de Sydney.

Las Tablas 1 y 2 resumen los valores de tasas de generación de viajes en auto obtenidos en algunos de los estudios antes citados. La Tabla 1 presenta valores de las horas pico de la mañana (Pico AM) y de la tarde (Pico PM), como así también valores diarios correspondientes al promedio de viajes en días de la semana (Prom. Sem.) entendiendo como días de la semana de lunes a viernes. La Tabla 2 presenta valores obtenidos en las franjas horarias indicadas.

En lo referente a antecedentes locales pueden citarse estudios orientados a estimar el impacto del ambiente construido sobre los viajes motorizados y no motorizados (Riera 2012, Riera y Brusa, 2012; Riera y Galarraga, 2013).

\section{ASPECTOS METODOLÓGICOS}

La metodología que se propone en este estudio incorpora las variables de movilidad urbana sustentable para estudiar la generación de viajes de unidades residenciales.

Tabla 1. Tasas de generación de viajes en auto según tipología de Emprendimiento [veh/un]

\begin{tabular}{|c|c|c|c|c|c|c|}
\hline \multirow[b]{2}{*}{$\begin{array}{c}\text { Tipología de } \\
\text { Emprendi-miento }\end{array}$} & \multirow[b]{2}{*}{$\begin{array}{c}\text { Single-Family } \\
\text { Detached Housing }\end{array}$} & \multicolumn{2}{|c|}{ Condominium/Townhouse } & \multicolumn{3}{|c|}{ Apartment } \\
\hline & & $\begin{array}{c}\text { Condominium } \\
\text { Townhouse }\end{array}$ & $\begin{array}{c}\text { High-Rise } \\
\text { (3 o más pisos) }\end{array}$ & Apartment & $\begin{array}{l}\text { High-Rise } \\
\text { (>10 pisos) }\end{array}$ & $\begin{array}{c}\text { Mid-Rise } \\
(3<\text { Pisos }<10)\end{array}$ \\
\hline Código ITE & 210 & 230 & 232 & 220 & 222 & 223 \\
\hline \multicolumn{7}{|c|}{ Instituto de Ingeniería de Transporte de los Estados Unidos (ITE, 2012) } \\
\hline Prom. Sem. & 9.52 & 5.81 & 4.18 & 6.65 & 4.20 & - \\
\hline Pico AM & 0.77 & 0.44 & 0.34 & 0.55 & 0.34 & 0.35 \\
\hline Pico PM & 1.02 & 0.52 & 0.38 & 0.67 & 0.40 & 0.44 \\
\hline \multicolumn{7}{|c|}{ Densificación de Ambientes Urbanos, California (KIMLEY-HORN et al, 2008) } \\
\hline Pico AM & - & 0.46 & 0.10 & - & 0.05 & 0.22 \\
\hline Pico PM & - & 0.41 & 0.17 & - & 0.07 & 0.28 \\
\hline \multicolumn{7}{|c|}{ Tasas de generación de viajes en Mérida, Venezuela (ANGULO Y GUERRERO, 2008) } \\
\hline Pico AM & - & - & - & - & - & 0.31 \\
\hline Pico PM & - & - & - & - & - & 0.38 \\
\hline
\end{tabular}


Tabla 2. Tasas de generación de viajes en auto según densidad y usos de suelo [veh/un]

\begin{tabular}{lccccc}
\hline \multicolumn{7}{c}{ Tasas de generación de viajes en Niterói, Brasil (NITTRANS, 2011) } \\
\hline \multirow{2}{*}{ Región } & Oceanica & Pendotiba & Centro & $\begin{array}{c}\text { Santa } \\
\text { Rosa }\end{array}$ & \multirow{2}{*}{ Icarí } \\
\hline Densidad & Baja & Baja & Media & Media & Alta \\
\hline Tipología & Casas aisladas & Casas aisladas & Variadas & Variadas & Pred. edificio \\
\hline Uso de suelo & Residencial & Residencial & Mixto & Mixto & Mixto \\
\hline 6h-20h & 6.06 & 5.19 & 0.81 & 1.38 & 1.58 \\
6h30-9h30 & 1.28 & 1.63 & 0.22 & 0.37 & 0.40 \\
17h-20h & 1.46 & 1.10 & 0.23 & 0.34 & 0.41 \\
\hline \multicolumn{7}{c}{ Condominios Residenciales Horizontales en Niterói, Brasil (GRIECO, 2010) } \\
\hline Densidad & Baja & \multicolumn{3}{c}{ Alta } \\
\hline Usos de Suelo & Residencial & & & Mixto \\
\hline 6h30-20h & 5.10 & & & 0.26 \\
6h30-9h & 1.16 & & & 0.34 \\
16h-19h & 1.17 &
\end{tabular}

Existe una estrecha interrelación entre el transporte y el uso del suelo. Es razonable pensar que una urbanización densa y de uso mixto, mantiene las distancias entre orígenes y destinos más cortas y vuelven más atractivos los viajes caminando o en bicicletas, modos de transporte más amigables con el ambiente. Por otro lado, mejor accesibilidad al transporte público, mayor cercanía al centro de negocios de la ciudad y un diseño de la ciudad más amigable al peatón, incentivan a usar menos el automóvil particular y a realizar más viajes en transporte público, bicicletas o a pie. De esta forma, cuando hablamos de variables de movilidad urbana sustentable nos referimos a aquellas que buscan caracterizar el entorno urbano: tipos y diversidad de usos de suelo, accesibilidad, densidad residencial, y diseño urbano. Existen en estas cuatro categorías, gran cantidad de indicadores a estudiar. En este caso, se optó por aquellos que, según los antecedentes examinados, poseen un uso generalizado en este tipo de estudios. Estos son detallados más adelante.

El estudio busca caracterizar dos ambientes bien diferenciados con sus tasas de generación por vivienda y por persona. Dentro de un nivel socio económico similar se estudian, por un lado, establecimientos residenciales de baja densidad, constituidos por casas aisladas y con escaso acceso al servicio de transporte público. Se incluyen en este grupo las tipologías residenciales conocidas como country y barrios cerrados, tipologías que contemplan casas unifamiliares en lotes individuales, con entrada y salida de vehículos controladas. Por otro lado, se estudian complejos de departamentos, con alta densidad, mejor acceso al servicio de transporte público y mayor diversidad de uso del suelo circundante. Se estudian aquí dos tipologías diferentes, los condominios y las torres de departamentos. Los condominios, están constituidos por construcciones de escasa altura, entre 3 y 4 pisos, agrupadas dentro de un mismo predio. Las torres de departamentos, contemplan una o más torres de más de 10 niveles de altura. En ambos casos, las construcciones se encuentran en un predio cerrado con control de ingreso.

En base a un estudio de casos en la ciudad de Córdoba, Argentina, se pretende identificar las diferencias en la generación de viajes para cada modalidad de transporte a fin de avanzar en la comprensión de ambientes de transporte más sustentables. Las variables explicativas relevadas se clasifican en cuatro grandes grupos: variables socioeconómicas, características del emprendimiento, variables relativas al entorno y características de los viajes.

Las variables socioeconómicas buscan determinar el nivel socioeconómico de las viviendas, a partir de estudiar cómo está conformado el grupo familiar: cantidad de personas y edades de los residentes, sexo, nivel educativo, ocupación, si poseen o no licencia de conducir, nivel de ingreso, número de vehículos por vivienda (autos, motos, bicicletas), cantidad de dormitorios que posee la vivienda.

Dentro de las características de los emprendimientos en este estudio se analiza además de la tipología, el número de viviendas ocupadas por emprendimiento.

La caracterización del entorno urbano se define a partir de indicadores pertenecientes a cuatro categorías: tipos y diversidad de usos de suelo, accesibilidad (localización, distancia en relación al centro, sistema de transporte público disponible), densidad residencial, y diseño urbano (caracterización de la red local de calles). Estos indicadores se estudian en un área de influencia de radio $500 \mathrm{~m}$. del acceso al establecimiento (distancia considerada razonable a recorrer a pie).

La diversidad de usos de suelo se ha medido en variados estudios utilizando una amplia gama de indicadores, desde simples recuentos hasta complejos índices. En este caso se optó por aplicar cuatro indicadores: la Entropía (Entrop), la Diversidad (Divers), el Índice de Simpson (Simp) y la Longitud de Interacción (Interac), ya sea por su uso generalizado en este tipo de estudios, por la facilidad en su definición, o por su capacidad para medir interacción o complementariedad entre usos de suelo.

La Entropía se definió en este estudio como:

$$
\text { Entropia }=-\sum \ln P_{i}\left(\frac{\ln P_{i}}{\ln n}\right)
$$

Donde "Pi" son las proporciones de cada una de las categorías de usos de suelo y " $n$ " es igual al número de categorías estudiadas. En esta caso, los usos de suelo relevados se clasificaron en siete categorías: residencial, comercial, educativo, salud, industrial, recreativo y otros.

Para el caso de la Diversidad (Rajamani et al., 2003), se modificó la ecuación de Rajamani cambiando el uso industrial por la suma de usos educativo y de salud, considerando en otros usos la suma de las categorías industrial, recreativo y otros.

$D i v=1-\left(\frac{\left(\frac{R e s}{T}-1 / 4\right)+\left(\frac{C o m}{T}-1 / 4\right)+\left(\frac{E d u+\text { Sal }}{T}-1 / 4\right)+\left(\frac{\text { Otro }}{T}-1 / 4\right)}{3 / 2}\right)$ 
En esta ecuación, se identifica "Res" como el área correspondiente a uso residencial, "Com" a uso comercial, "Edu" y "Sal", a educativos y de salud respectivamente, "Otro" a otro uso no considerado en las anteriores categorías, mientras que la variable "T" corresponde a la suma de todas estas áreas. Un valor de 0 para esta medida significa que el uso de suelo en el área bajo estudio es homogéneo (un único uso de suelo), mientras que un valor de 1 indica perfecta mezcla de los cuatro usos de suelo.

El Índice de Simpson se definió como:

$$
\text { Indice de Simpsons }=1-\sum P_{i}^{2}
$$

Donde "Pi" es la proporción del área de cada una de las siete categorías de uso de suelo relevadas.

Finalmente, la Longitud de Interacción, se definió considerando los bordes de interacción entre polígonos de usos Residencial con aquellos usos que se consideró pueden existir propósitos de viaje posibles. Estos son: uso comercial, educativo, de salud y recreativo.

Entre los indicadores para medir la accesibilidad, se encuentran la distancia al centro de negocios de la ciudad, ya sea medida en línea recta, o bien por ruta vehicular, la distancia a la parada de transporte público más cercana al polo generador en estudio, el número de líneas que sirven dicha parada, como así también la cantidad de paradas y líneas de transporte público en el área de influencia.

La densidad se define como el cociente de la población del área en estudio a la superficie de dicha área. La población de la zona de influencia se determinó a partir de los datos del censo realizado por la Dirección General de Estadísticas y Censos de la Provincia de Córdoba en toda la Provincia en el año 2008, y aplicando a la población obtenida, una tasa de crecimiento anual del $0,4 \%$ a fin de obtener la población al año en curso.

El diseño urbano hace referencia a la caracterización de la red local de las calles. Estos indicadores buscan capturar la adecuación de las calles del barrio para el uso de peatones y bicicletas. Los indicadores estudiados en este caso fueron la cantidad de manzanas, el número de nodos, la cantidad de cul de sacs y la longitud de enlaces en el área de influencia.

Las variables relevadas para caracterizar los viajes fueron el número de viajes generados con origen o destino en el hogar, la modalidad empleada y el motivo de los viajes. En cuanto a la cantidad de viajes generados, se estudiaron los viajes producidos y los atraídos por cada uno de los emprendimientos en las horas pico, como así también, los generados por unidad de vivienda para un día completo. Todos estos viajes corresponden a días de semana.

La obtención de las variables descriptas se concretó mediante el estudio de planos e imágenes satelitales disponibles, relevamientos in situ, entrevistas con los desarrollistas y administradores de los sitios bajo estudio, como así también a través de conteos manuales de vehículos en los ingresos a los emprendimientos y encuestas a los residentes. Entre los planos disponibles, se encuentran planos de transporte público de pasajeros de la Municipalidad de Córdoba, y los planos correspondientes al servidor Google Maps. Cabe destacar el uso de imágenes satelitales y del reciente servicio de imágenes panorámicas de Google, Street View, disponible desde septiembre de 2014 para la ciudad de Córdoba, que permite recorrer la mayoría de las calles de la ciudad con imágenes panorámicas a nivel de calle en $360^{\circ}$ de movimiento horizontal y $290^{\circ}$ de movimiento vertical. Esta resultó una herramienta muy potente y útil para completar los relevamientos realizados in situ.

En cuanto a las características de los conteos, y siguiendo los lineamientos propuestos por el Trip Generation Handbook (2001), se realizaron conteos de volúmenes de tránsito direccionales (entrando y saliendo del desarrollo) fraccionados en períodos de 15 minutos durante los períodos pico de la mañana y de la tarde del polo generador. Los conteos fueron realizados días de semana, entre martes y jueves, de forma manual, en los accesos a los establecimientos y permitieron determinar también la modalidad de los mismos.

Con respecto a la encuesta, fue desarrollada en formato papel y en formato digital para ser respondida ingresando a Internet a fin de llegar a la gente por diferentes vías de comunicación, logrando un mayor número de respuestas. El formulario desarrollado para la encuesta consta de cuatro secciones: la primera sección busca recolectar datos con respecto a la vivienda. Busca conocer la cantidad de personas que viven en el hogar, dormitorios que posee, y vehículos disponibles, distinguiendo entre automóviles (autos/camionetas), motos y bicicletas. La sección dos, datos con respecto a las personas. Se busca conocer características de tipo demográficas del hogar. Para ellos se pregunta sobre Edad, Sexo, Nivel Educativo, Ocupación de cada uno de los integrantes del hogar y si poseen o no Licencia de Conducir. Se pregunta también si en el día correspondiente al registro de viajes alguna persona ajena al hogar viajo hacia y/o desde la vivienda. La sección tres recolecta datos con respecto a los viajes (sentido del viaje, hora, modo y motivo). Se solicita anotar los datos con respecto a los viajes realizados por todas las personas que habitan la vivienda como así también por personas ajenas a la misma durante un día de semana (martes, miércoles o jueves) completo (de 0 a 24 horas). Finalmente, la sección cuatro, de respuesta opcional, se refiere a los ingresos del hogar. Se desea conocer el rango en que se encuentran los ingresos totales del hogar.

\section{ESTUDIO DE CASOS}

Como se mencionara anteriormente, esta investigación se centra en el estudio de casos en la ciudad de Córdoba, Argentina. La ciudad de Córdoba se ha ido expandiendo cada vez más en búsqueda de tierra más barata y mejores condiciones de habitabilidad, conformándose así nuevos loteos tales como country y barrios cerrados. Estas nuevas urbanizaciones asentadas a las afueras de la ciudad en general no poseen un buen servicio de transporte urbano de pasajeros, ya que la falta de conectividad obliga a que se alarguen los recorridos y esto dificulta notablemente la prestación del mismo, con el aumento consecuente de sus costos y sin una demanda suficiente para satisfacer dichos gastos. Por otro lado, en las zonas más próximas al centro de la ciudad, debido a los elevados costos de la tierra en estos sectores, se tiende al desarrollo de emprendimientos en altura, conformándose así emprendimientos tales como condominios residenciales o barrios cerrados en altura. 


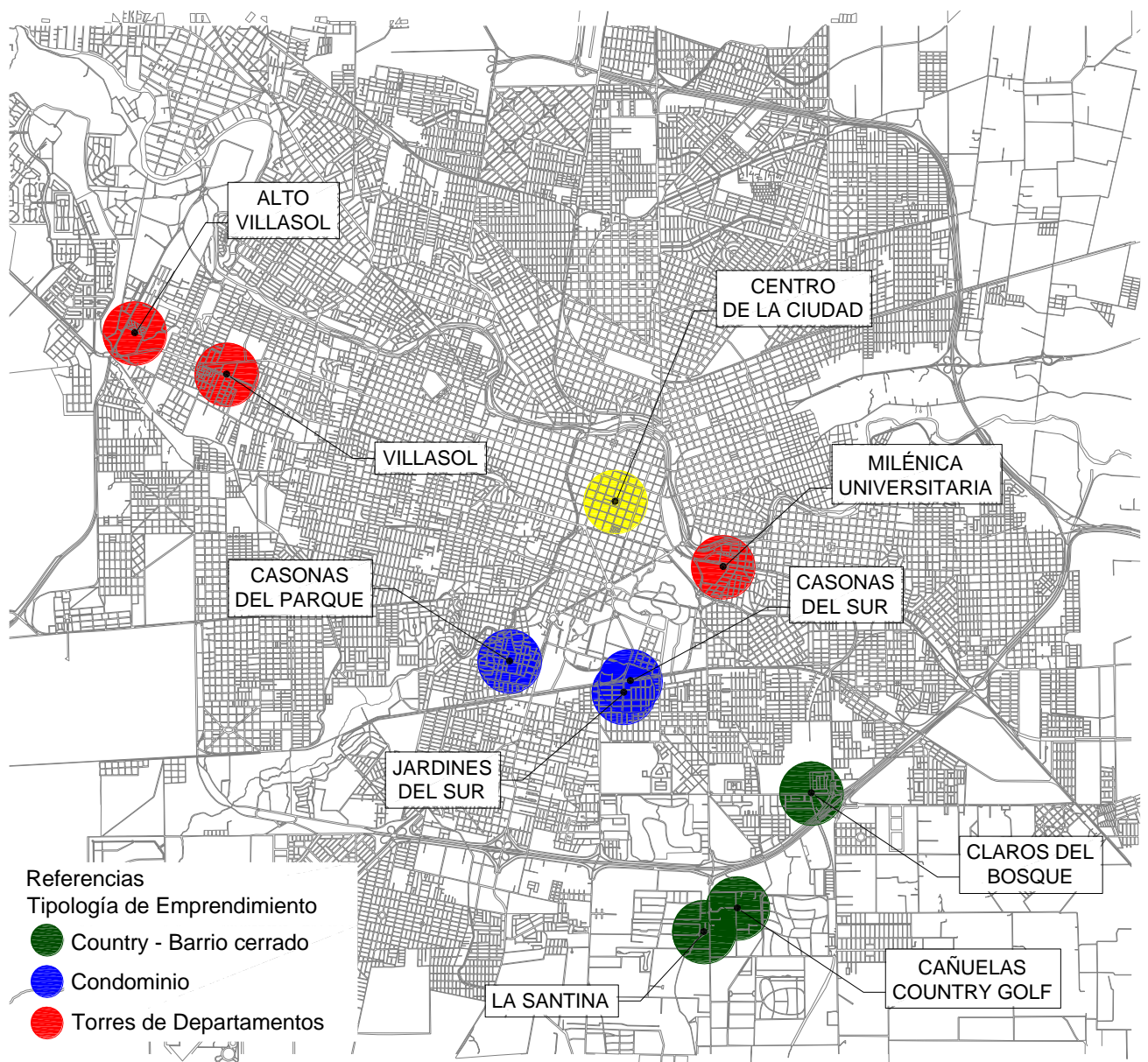

Figura 1. Ubicación de los Emprendimientos Residenciales Estudiados

Estos poseen generalmente un mejor servicio de transporte urbano de pasajeros y suelen ubicarse en zonas con usos de suelo mixto.

A fin de caracterizar estos dos ambientes bien diferenciados, se procedió a relevar algunos de los principales emprendimientos residenciales de la ciudad, seleccionándolos de acuerdo a los siguientes criterios.

Entre las características contempladas al momento de optar por los emprendimientos se encuentran: nivel socioeconómico similar entre los diferentes emprendimientos, ubicación en zonas de densidad de población alta y baja, usos de suelo puramente residenciales y usos mixtos, sectores con servicio de transporte urbano de pasajeros buenos y servicios escasos. En cuanto a la muestra, se planteó estu- diar al menos tres desarrollos por cada tipología de establecimiento residencial. Cada desarrollo a elegir debe tener una ocupación razonablemente alta (al menos $85 \%$ ), y ser maduro (al menos 2 años de antigüedad) y estar localizado también en un área de desarrollo estabilizado, con acceso único o por entradas limitadas, y permiso de propietario o administrador para realizar los relevamientos. Bajo estas condiciones, los emprendimientos seleccionados para el estudio se muestran en la Figura 1.

Las Tablas 3 y 4 ofrecen los valores de los distintos indicadores obtenidos como resultado de los relevamientos del entorno urbano. Por otro lado, se obtuvieron datos acerca de los viajes generados a pie y en auto en horas pico

Tabla 3. Número de Unidades de vivienda e Indicadores de Usos de Suelo y Ubicación

\begin{tabular}{|c|c|c|c|c|c|c|c|c|c|}
\hline \multirow[t]{2}{*}{ Tipología } & \multirow{2}{*}{$\begin{array}{c}\text { Emprendimento } \\
\text { Residencial }\end{array}$} & \multirow[t]{2}{*}{ Unidades } & \multirow{2}{*}{$\begin{array}{l}\text { Densidad } \\
\text { [Pob/Ha] }\end{array}$} & \multicolumn{4}{|c|}{ Indicadores de usos de suelo } & \multicolumn{2}{|c|}{$\begin{array}{l}\text { Distancia }[\mathrm{Km}] \\
\text { Centro }\end{array}$} \\
\hline & & & & Divers. & Entrop. & Simp. & Interac. & Auto & Recta \\
\hline \multirow{3}{*}{ Condominio } & Casonas Parque & 96 & 56.53 & 0.29 & 0.44 & 0.38 & 4477 & 3.70 & 3.00 \\
\hline & Casonas Sur & 217 & 50.78 & 0.34 & 0.46 & 0.42 & 4497 & 3.00 & 2.85 \\
\hline & Jardines Sur & 48 & 64.74 & 0.33 & 0.53 & 0.42 & 5924 & 3.20 & 3.05 \\
\hline \multirow{3}{*}{ Country } & Claros Bosque & 210 & 19.62 & 0.35 & 0.50 & 0.53 & 38 & 7.30 & 5.55 \\
\hline & La Santina & 56 & 3.26 & 0.33 & 0.57 & 0.41 & 1539 & 7.80 & 7.00 \\
\hline & Cañuelas & 90 & 3.46 & 0.34 & 0.38 & 0.41 & 205 & 7.80 & 6.75 \\
\hline \multirow{3}{*}{ Edificio } & Milenica & 520 & 54.07 & 0.53 & 0.70 & 0.60 & 2926 & 2.20 & 2.05 \\
\hline & Villasol & 834 & 39.05 & 0.69 & 0.76 & 0.69 & 1544 & 6.40 & 6.15 \\
\hline & Alto Villasol & 989 & 20.40 & 0.66 & 0.71 & 0.66 & 1000 & 8.30 & 7.55 \\
\hline
\end{tabular}


GALARRAGA, J.J.; BORDESE, L.

Tabla 4. Indicadores de Diseño Urbano y Accesibilidad

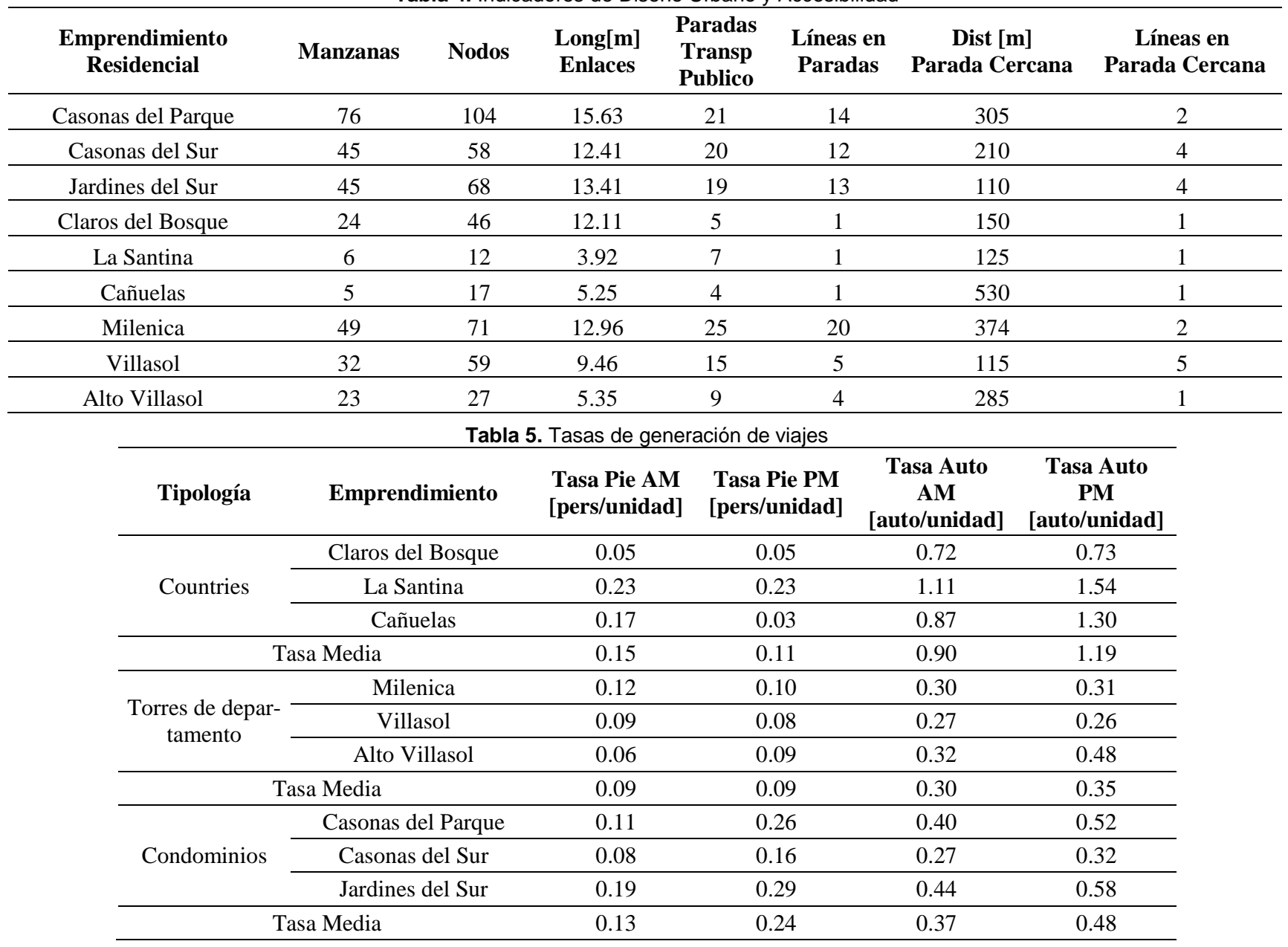

de la mañana y de la tarde por cada uno de los emprendimientos. Y a partir de estos, las tasas de generación de cada uno y tasas medias por tipología, según se muestra en la Tabla 5 .

Comparando estos valores con los valores del ITE, según se muestra en la Tabla 6, puede observarse que para el caso de los countries, comparables con el uso de suelo correspondiente al código 210 del ITE (Vivienda unifamiliar), las tasas medias observadas son $17 \%$ superiores, tanto en horas pico de la mañana como de la tarde. Por el contrario, para las tipologías de mayor densidad, se tienen tasas de generación menores a las tasas del ITE, con un promedio del $13 \%$ menos.Finalmente, la Tabla 7, relaciona las tasas de generación de viajes en auto de los emprendimientos de baja densidad, con los de alta densidad, tanto para los valores observados como para los valores del ITE. Esta relación muestra que la tasa de los countries es cercana a 3 veces mayor que la de los condominios y torres de departamentos en cuanto a los valores observados, mientras que en el caso de los valores del ITE, esta relación es más cercana a 2.

Con respecto a los relevamientos de datos mediante el uso de encuestas, cabe destacar una serie de dificultades enfrentadas que hicieron imposible la recolección de una muestra estadísticamente representativa. Entre estas dificultades, se destaca la falta de interés y las dificultades para comprender y responder las encuestas sin la ayuda de un encuestador de las personas que recibieron la encuesta por mail o bien junto con las expensas.

Tabla 6. Comparación entre Tasas Medias de Generación en Auto observadas y tasas del ITE

\begin{tabular}{cccccccc}
\hline Tipología & \multicolumn{2}{c}{ Tasas Observadas } & \multicolumn{3}{c}{ Tasas ITE } & \multicolumn{3}{c}{ Comparación ITE } \\
\cline { 2 - 8 } Emprendimiento & Pico AM & Pico PM & Código & Pico AM & Pico PM & Pico AM & Pico PM \\
\hline Country & 0.90 & 1.19 & 210 & 0.77 & 1.02 & $-17 \%$ & $-17 \%$ \\
\hline Departamentos & 0.30 & 0.35 & 222 & 0.34 & 0.40 & $13 \%$ & $12 \%$ \\
\hline Condominio & 0.37 & 0.48 & 230 & 0.44 & 0.52 & $17 \%$ & $9 \%$
\end{tabular}

Tabla 7. Relación entre tasas de generación en auto en emprendimientos de baja y alta densidad Relación de tasas Country/Departamentos

Relación de tasas Country/Condominio

\begin{tabular}{lcccccc}
\hline & Observadas & ITE & Comparación & Observadas & ITE & Comparación \\
\hline Pico AM & 3.02 & 2.26 & $-33 \%$ & 2.45 & 1.75 & $-40 \%$ \\
\hline Pico PM & 3.39 & 2.55 & $-33 \%$ & 2.5 & 1.96 & 1.86 \\
\hline Promedio & 3.2 & 2.41 & $-33 \%$ & 2.47 & $-33 \%$ & \\
\hline
\end{tabular}


Tabla 8. Resultados de las encuestas realizadas en el hogar

\begin{tabular}{lccc}
\hline & $\begin{array}{c}\text { Torres de deptos } \\
\text { Alto Villasol }\end{array}$ & $\begin{array}{c}\text { Condominio } \\
\text { Casonas Parque }\end{array}$ & $\begin{array}{c}\text { Country } \\
\text { Claros del Bosque }\end{array}$ \\
\hline Hogares encuestados & 43 & 31 & 42 \\
\hline Promedio Personas H por Hogar & 2.21 & 2.32 & 3.45 \\
\hline Promedio Personas por Hogar (inc. Ajenas) & 3.05 & 2.71 & 4.02 \\
\hline Promedio de Autos por Hogar & 1.12 & 1.10 & 1.57 \\
\hline Cantidad de Hogares con auto & $91 \%$ & $84 \%$ & $100 \%$ \\
\hline Viajes en Modos Sustentables & $28 \%$ & $18 \%$ & $12 \%$ \\
\hline Viajes Modos Sustentables por hogar & 2.07 & 1.29 & 5.26 \\
\hline Viajes diarios en auto por hogar & 3.51 & 3.65 & 1.52 \\
\hline Viajes diarios en auto por persona & 1.59 & 1.57 & 3.35 \\
\hline Viajes diarios en auto por vehículo & 3.15 & 3.32 & \\
\hline
\end{tabular}

En este primer relevamiento, se obtuvo un porcentaje muy bajo de respuestas que hizo rever el método de recolección de los datos. Luego, se decidió realizarlas personalmente, y surgió entonces una nueva dificultad, la obtención de los permisos para ingresar a los emprendimientos. Es por ello que, si bien se había planteado inicialmente realizar las encuestas en todos los emprendimientos seleccionados y con un objetivo de lograr un número de encuestas estadísticamente representativo, se decidió realizar las encuestas en un emprendimiento de cada tipología, obteniendo una muestra mínima deseada de 40 encuestas, o un mínimo absoluto de 30 encuestas.

La Tabla 8 resume los principales resultados obtenidos de las encuestas realizadas. Por otro lado, la Figura 2 detalla cómo se distribuyen los viajes relevados en las encuestas según los distintos modos de transporte, en tanto la Figura 3 muestra la distribución según los motivos de viaje.

Comparando los valores de tasas de generación de viajes en auto diarias obtenidas de las encuestas con las tasas del ITE, se desprende que las tasas diarias reportadas son muy inferiores a las tasas del ITE.

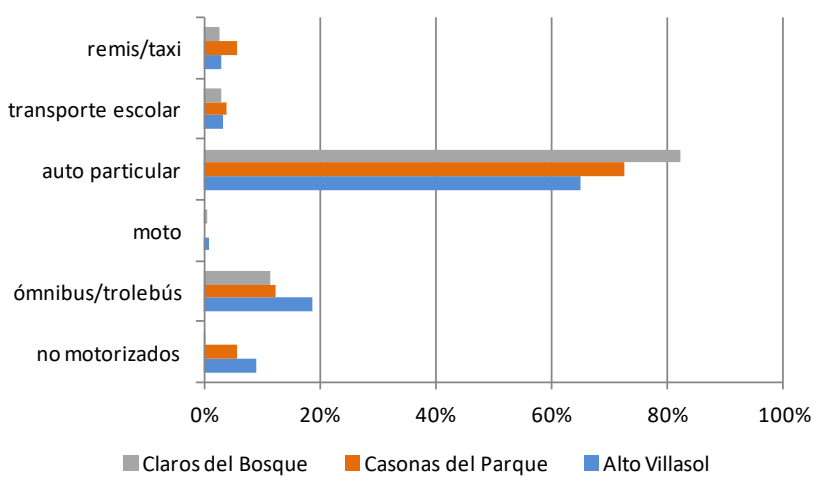

Figura 2. Distribución de los viajes según los modos

Por otro lado, se puede observar que existe una alta dependencia del uso del automóvil en los countries, donde todos los hogares poseen al menos un auto, y donde el porcentaje de viajes en modos sustentables (a pie, en bicicleta o en ómnibus) es mucho menor que en los emprendimientos ubicados en sectores de mayor densidad y diversidad de usos de suelo.

En cuanto a las tasas de generación en auto obtenidas, cabe destacar la gran semejanza de las tasas por personas y por vehículos para los tres emprendimientos, no así en las tasas por hogar, donde la tasa del country es mucho mayor.
Esto es explicado por el mayor promedio de personas que habitan en los hogares ubicados en esta tipología, donde se asientan generalmente familias con hijos en edad escolar. Por el contrario, en los departamentos y condominios, viven en su mayoría, parejas jóvenes sin niños o con un hijo pequeño, parejas mayores o adultos solos.

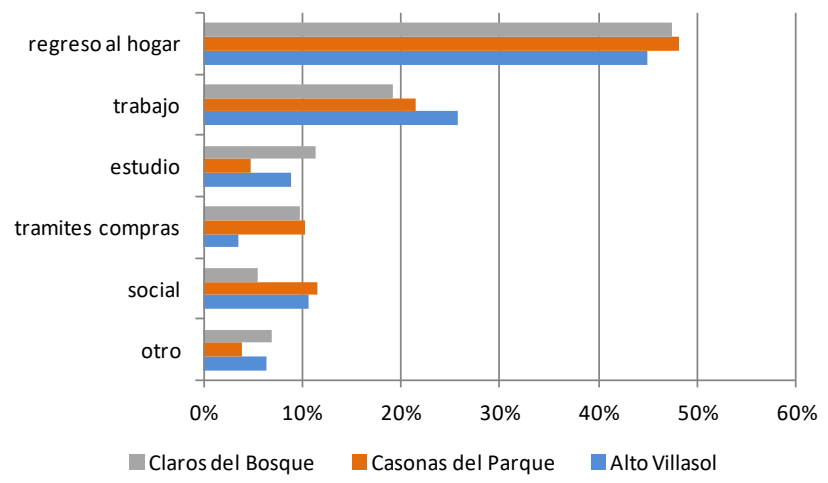

Figura 3. Distribución de los viajes según el motivo

\section{CARACTERIZACIÓN DE LOS VIAJES Y FORMULACIÓN DE MODELOS DE GENERACIÓN}

Se estudian los viajes generados, como la suma de los viajes producidos más los atraídos por cada uno de los emprendimientos, es decir, aquellos viajes que tienen su origen o destino en los hogares estudiados. A diferencia de la metodología propuesta en el manual del ITE, se estudian modelos generales, abarcando todas las tipologías de emprendimientos e incluyendo, además de la variable unidades de vivienda, variables relativas al entorno. Se plantean modelos para los viajes generados en auto, como así también para los generados a pie. Se presentan a continuación los modelos que mejor logran explicar la generación de los viajes, sin llegar a ser demasiado complejos.

Los indicadores utilizados para la elección de los modelos reportados fueron, además del coeficiente R2 corregido, la suma de cuadrados de residuos y la desviación típica de la regresión, criterios de decisión de Akaike, Schwarz y Hannan-Quinn, niveles de significancia y desviación típica de las variables independientes. Se realizaron las pruebas de no colinealidad entre las variables independientes, de normalidad de los residuos, de homocedasticidad.

En el caso de los viajes generados en auto, se optó por presentar la ecuación ajustada pasando por el origen, ya 
que al incorporar al modelo el término independiente, éste tomaba un valor negativo, pequeño, y no significativo.

$$
\begin{array}{ll}
\text { Auto } A M=0.303 \text { Unidades }+61.1 \text { Dcountry } & \mathrm{R}^{2}=0.99 \\
\text { Auto } P M=0.378 \text { Unidades }+74.1 \text { Dcountry } & \mathrm{R}^{2}=0.94 \\
\text { Pie } A M=6.548+0.070 \text { Unidades } & \mathrm{R}^{2}=0.86 \\
\text { PiePM }=0.0893 \text { Unidades }+13.9 \text { Dcondominio } & \mathrm{R}^{2}=0.99 \\
\text { PiePM }=15.568+0.0712 \text { Unidades }-15.0197 \text { Dcountry } & \mathrm{R}^{2}=0.97
\end{array}
$$

Para los viajes a pie en horas pico de la tarde, el modelo sin término independiente muestra su mayor participación para los Condominios, en tanto que el modelo con término independiente indica su menor participación en los countries. Se reportan ambos modelos para informar al respecto.

Como puede observarse, los modelos a los que se arribó no poseen variables relativas al entorno, incorporando, además del número de unidades de vivienda, variables de tipo dummy que permiten definir la tipología de emprendimiento. Para el caso de los modelos de generación de viajes en auto, resulta importante definir si el emprendimiento en estudio es country o no.

Para el caso de los viajes a pie en horas pico de la mañana, el tipo de emprendimiento no resulta significativo. En los viajes a pie en horas pico de la tarde importa definir si se trata de condominios. También se obtienen buenos resultados al diferenciar el tipo de emprendimiento country.

Por otro lado, surgen del proceso de modelación algunos modelos que incluyen variables relativas al entorno, que permiten arribar también a buenas estimaciones, a la vez que permiten comprender el efecto de estos factores sobre la generación de viajes. Para los viajes en auto, se obtuvieron buenos modelos incluyendo la variable diversidad, mientras que para viajes a pie se desarrollaron modelos con buenos resultados incluyendo la variable interacción.

$$
\begin{array}{ll}
\text { AutoAM }=95.216+0.448 \text { Un }-339.736 \text { Div }+63.949 \text { Dcountry } & \mathrm{R}^{2}=0.98 \\
\text { AutoPM }=300.819+0.855 \text { Un }-1096.76 \text { Div }+89.011 \text { Dcountry } & \mathrm{R}^{2}=0.93 \\
\text { PieAM }=2.089+0.0612 \text { Un }+0.00803 \text { Inter. }-36.981 \text { Dcond. } & \mathrm{R}^{2}=0.94 \\
\text { PiePM }=-3.373+0.0875 \text { Un }+0.00341 \text { Inter. } & \mathrm{R}^{2}=0.97
\end{array}
$$

Para el caso de los viajes a pie, los datos de la variable interacción presentan valores medios bajos para countries, intermedios para departamentos y altos para condominios. Su inclusión modifica apreciablemente la estructura de los modelos. Las aplicaciones de los mismos deberían restringirse al entorno de datos usados para su calibración, del orden de 50 a $1500 \mathrm{~m}$ para countries, de 1000 a $3000 \mathrm{~m}$ para departamentos y de 4500 a $6000 \mathrm{~m}$ para condominios.

Finalmente, se estudian los viajes totales producidos durante las horas pico, relacionándolos con la variable unidades de vivienda, como así también con variables relativas al entorno. Se define a los viajes producidos totales como la suma de los viajes con origen en cada uno de los emprendimientos en la hora pico de la mañana más los viajes con origen en cada uno de ellos en la hora pico de la tarde. Se estudian tanto los viajes producidos a pie como los producidos en auto.

En la Figura 4, con la cantidad de unidades en abscisas y el número de viajes producidos en ordenadas, se muestra la relación entre ambas variables y la aproximación mediante una regresión lineal simple.

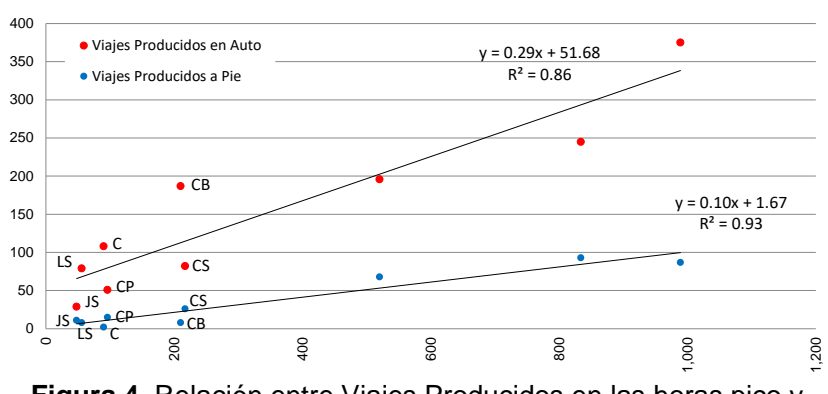

Figura 4. Relación entre Viajes Producidos en las horas pico y Unidades de Vivienda

Al estudiarlo en detalle, puede observarse que, para un número de unidades de vivienda similares en un emprendimiento tipo Condominio y en uno tipo Country, se da que los viajes producidos en auto van por encima de la regresión lineal y los producidos a pie por debajo de la misma para el country, mientras que sucede al contrario en el condominio. Es decir, para un número similar de unidades de viviendas, los viajes en auto en el country son mayores, mientras que los viajes a pie son más en el condominio. A fin de poder visualizarlo en el gráfico, se han colocado las siglas de cada emprendimiento junto a las observaciones. Para los countries se tiene, La Santina (LS), Cañuelas (C) y Claros del Bosque (CB), y para los condominios, Jardines del Sur (JS), Casonas del Parque (CP) y Casonas del Sur (CS).

$\mathrm{Al}$ igual que al estudiar los viajes generados, puede observarse que contemplando las variables explicativas unidades de vivienda y dummy que permiten definir la tipología de emprendimiento se logra una buena inferencia. Las variables dummy a incorporar son las que permiten definir las tipologías departamentos y condominios en el caso de los viajes producidos a pie, mientras que para los viajes producidos en auto, la tipología a diferenciar es la country.

$$
\begin{array}{ll}
\text { ProducidosPie }=0.334+0.0477 \text { Un }+11.254 \text { Dcondom }+45.0425 \text { Ddepto } & \mathrm{R}^{2}=0.99(13) \\
\text { ProducidosAuto }=10,7+0,338 \text { Un }+73,9 \text { Dcountry } & \mathrm{R}^{2}=0.95(14)
\end{array}
$$

Para el caso de viajes producidos, la incorporación de variables relativas al entorno mejora la estimación de los viajes. Sin embargo, dada la complejidad de la obtención de estas variables, se consideran más útiles los modelos anteriores.

$$
\begin{array}{ll}
\text { Prod. } \text { Pie }=-2,54+0,0598 \text { Un }+0,00254 \text { Inter }+33,9 \text { Ddepto } & \mathrm{R}^{2}=0.99(15) \\
\text { Prod. Auto }=163+0,569 \text { Un }-543 \text { Divers }+77,9 \text { Dcountry } & \mathrm{R}^{2}=0.96(16)
\end{array}
$$

Las variables relativas al entorno que resultaron más significativas fueron aquellas que definen usos de suelo: la 
interacción para los viajes producidos a pie y la diversidad para los producidos en auto.

\section{CONCLUSIONES}

El proyecto apunta a resolver una necesidad creciente de generar acciones orientadas a promover una movilidad urbana sustentable. Para ello resulta necesario disponer de datos y herramientas de análisis aplicables al tránsito y las actividades urbanas.

La estimación de tasas y modelos de generación permite cuantificar adecuadamente el impacto de emprendimientos residenciales sobre la movilidad urbana, convirtiéndose en una importante herramienta para la planificación.

Las ciudades argentinas carecen en general de estudios para la determinación de tasas de generación. La utilización de tasas de generación de otros países pueden producir estimaciones erróneas, dando lugar a infraestructuras subdimensionadas o sobredimensionadas.

A partir del estudio de 9 emprendimientos de tres tipologías residenciales diferentes y con distintas características en su entorno urbano, se ha logrado caracterizar tipos diferentes de unidades residenciales. Se han obtenido tasas de generación de viajes en auto y a pie, y se han formulado modelos.

Las tasas y modelos obtenidos revelan indicios de que los factores de localización y entorno urbano influyen en la movilidad.

Las tasas de generación de viajes por hogar obtenidas en horas pico de la mañana y de la tarde, muestran grandes diferencias entre los dos ambientes estudiados y con las tasas del ITE, lo que afirma la necesidad de realizar estudios locales.

Las tasas de generación de viajes en auto obtenidas para los emprendimientos residenciales de baja densidad, constituidos por casas aisladas y con escaso acceso al servicio de transporte público triplican los valores observados en complejos de departamentos, con alta densidad, mejor acceso al servicio de transporte público y mayor diversidad de uso del suelo circundante. Esto lleva a pensar en la necesidad de políticas de planificación territorial en post de reducir las necesidades de desplazamiento limitando la expansión urbana y tendiendo a ciudades más compactas, con usos de suelo mezclados, en las que el acceso a los bienes y servicios se encuentra dentro de un entorno reducido, lo que lleva a hacer más atractivos los viajes a pie, en bicicleta o en transporte público, modos de transporte más amigables con el ambiente.

Del análisis de los modelos obtenidos surge que las principales variables explicativas de los viajes generados son el número de unidades de vivienda ocupadas y la tipología de emprendimiento.

Algunas variables asociadas a la diversidad y complementariedad de usos de suelo, tal como la diversidad y la interacción, también mostraron una buena correlación con la generación de viajes, permitiendo mejorar en algunos casos la bondad de predicción de los modelos correspondientes. Las variables de diseño urbano y accesibilidad al transporte público no mostraron en general buena correlación con los viajes generados.
Los resultados obtenidos de las encuestas en hogares revelan la dependencia en el uso del automóvil de las familias que viven en countries, donde todos los hogares poseen al menos un auto, y donde el porcentaje de viajes en modos sustentables (a pie, en bicicleta o en ómnibus) es mucho menor que en los emprendimientos ubicados en sectores de mayor densidad y diversidad de usos de suelo.

Los modelos obtenidos representan un primer intento de modelado de viajes. Por lo tanto, se deben utilizar estos modelos con precaución, dentro de los márgenes de unidades de viviendas y para niveles socioeconómicos como características de entorno similares, ya que de lo contrario pueden generar resultados inexactos.

Entre las recomendaciones para futuras investigaciones, se considera importante incorporar un mayor número de emprendimientos al estudio para la prueba efectiva de los resultados. En cuanto a los conteos, y existiendo en general registros de entradas y salidas por parte de las guardias de los emprendimientos, resultaría de gran ayuda contar con la colaboración de quienes administran los emprendimientos a fin de obtener mejor y mayor cantidad de información.

\section{REFERÊNCIAS}

ANGULO, R y GUERRERO, G (2008). Determinación de indices de generación de viajes para conjuntos residenciales de la ciudad de Mérida. Escuela de Ingeniería Civil, Facultad de Ingeniería, Universidad de los Andes, Mérida, Venezuela.

ARRINGTON, G. B. y CERVERO, R. (2008). Vehicle Trip Reduction Impacts of Transit-Oriented Housing. Journal of Public Transportation, Vol. 11, No. 3. DOI: 10.5038/23750901.11.3.1

GORI, S.; NIGRO, M.; PETRELLI, M. (2013) Land use and public transport interaction, World Conference on Transport Research WCTR XIII. Rio de Janeiro, Brasil.

GRIECO, E. P. (2010). Taxas de Geração de Viagens em Condomínios Residenciais - Niterói - Estudo de Caso, Rio de Janeiro. Universidade Federal do Rio de Janeiro, Brasil.

HANDY, S.; CAO, X. y MOKHTARIAN, P. (2005). Correlation or causality between the built environment and travel behavior? Evidence from Northern California. Transportation Research Part D: Transport and Environment 10 (6), 427-444. DOI: 10.1016/j.trd.2005.05.002

HANDY, S.; SHAFIZADEH, K. y SCHNEIDER, R. (2013). California Smart-Growth Trip Generation Rates Study. University of California, Davis for the California Department of Transportation. Disponible en:

http://downloads.ice.ucdavis.edu/ultrans/smartgrowthtripgen/Fin al_Report.pdf - Acceso 17/10/2016

KHATTAK, A. J. y RODRIGUEZ, D (2005). Travel Behavior in Neo-Traditional Neighborhood Developments: A Case Study in USA. Transportation Research Part A 39. 481-500. DOI: 10.1016/j.tra.2005.02.009

KIMLEY-HORN AND ASSOCIATES, INC. (2008). TripGeneration Rates for Urban Infill Land Uses in California. Prepared for The California Department of Transportation (Caltrans), by Association of Bay Area Governments (ABAG). Disponible en:

http://www.dot.ca.gov/newtech/researchreports/reports/2008/ca_i nfill_trip_ratesphase_1_final_report_appendices_4-24-08.pdf Acceso 17/10/2016 
LA PAIX, L.; MONZÓN, A. y CHERCHI, E. (2012) Modelling the relationship between Urban Environment and Travel Behavior: Policy and Indicators. International Scientific Conference on Mobility and Transport. Disponible en: http://doc.utwente.nl/84980/1/La_Paix_et_al._Modeling_the_rela tionship_between_urban_env.pdf - Acceso 17/10/2016

NITTRANS. (2011) Niteroi, Transporte e Transito. Caderno Técnico 01/2011.

ORTÚZAR, J y WILLUMSEN, L, (2011) Modelling Transport. John Wiley and Sons.

PORTUGAL L. S. (Org.) (2012) Polos Geradores de Viagens Orientados a Qualidade de Vida e Ambiental: Modelos e Taxas de Geracao de Viagens, Editora Interciencia, Rio de Janeiro.

PORTUGAL, L. S.; FLOREZ, J.; SILVA, A. N. R. (2010) Rede de Pesquisa em Transportes: Um Instrumento de Transformacao e Melhora da Qualidade de Vida, Transporte, vol XVIII, $n^{\circ}$ 1, p.616. DOI: 10.14295/transportes.v18i1.395

PORTUGAL, L. S. y GOLDNER, L. G., (2003) Estudo de Polos Geradores de Tráfego e de seus Impactos nos Sistemas Viários e de Transportes. $1^{\circ}$ Edição. Editora Edgard Blucher Ltda. São Paulo.

RAJAMANI, J., C. R. BHAT, HANDY, S., KNAAP G., SONG Y. (2003). Assessing impact of urban form measures on nonwork trip mode choice after controlling for demographic and level-ofservice effects. Transportation Research Record: Journal of the Transportation Research Board 1831: 158-165. DOI: $10.3141 / 1831-18$

RIERA, A. (2012). Estudio de las Perspectivas del Transporte No Motorizado en Ciudades Argentinas Aplicando Modelos de Generación de Viajes. Tesis de Maestría en Transporte. UNC. Córdoba, Argentina

RIERA, A. y BRUSA, M. J. (2012). Impacto Del Ambiente Construido Sobre Los Viajes Motorizados. XVII Congreso Panamericano de Ingeniería de Tránsito, Transporte y Logística. Santiago. Chile. Disponible en:

http://redpgv.coppe.ufrj.br/index.php/es/produccion/articuloscientificos/2012-1/704-impacto-del-ambiente-construido-sobrelos-viajes-motorizados-panam-2012/file - Acceso 17/10/2016

RIERA, A. y GALARRAGA, J. (2013). Influencia del ambiente construido en la Generación de viajes a pie en la ciudad de Córdoba. ANPET XXVII. Belém, Brasil. Disponible en: http://www.anpet.org.br/ssat/interface/content/autor/trabalhos/pu blicacao/2013/57_AC.pdf - Acceso 17/10/2016

TSAI, C.; MULLEY C. y CLIFTON G. (2012). The spatial interactions between public transport demand and land use characteristics in the Sydney Greater Metropolitan Area. Road \& Transport Research. Vol 21 No 4, pp 62-73. Disponible en: http://atrf.info/papers/2012/2012_Tsai_Mulley_Clifton.pdf Acceso 17/10/2016 